\title{
A bounded version of bosonic creation and annihilation operators and their related quasi-coherent states
}

\author{
F. Bagarello \\ Dipartimento di Metodi e Modelli Matematici, Facoltà di Ingegneria, \\ Università di Palermo, I - 90128 Palermo, Italy \\ E-mail: bagarell@unipa.it \\ home page: www.unipa.it $\backslash$ bagarell
}

\begin{abstract}
Coherent states are usually defined as eigenstates of an unbounded operator, the so-called annihilation operator. We propose here possible constructions of quasi-coherent states, which turn out to be quasi eigenstate of a bounded operator related to an annihilation-like operator. We use this bounded operator to construct a sort of modified harmonic oscillator and we analyze the dynamics of this oscillator from an algebraic point of view.
\end{abstract}




\section{Introduction}

One of the very few undergraduate examples of quantum mechanical systems for which a complete solution is known, the harmonic oscillator, suggests the introduction of the so-called ladder operators, the annihilation and creation operators $a$ and $a^{\dagger}$. They turn out to obey the commutation rule $\left[a, a^{\dagger}\right]=\mathcal{I}$ which implies that they are unbounded operators so that, for instance, they are not everywhere defined. Exactly the same kind of operators appear also in the analysis of a rather different system, a gas of bosons, and they are also almost everywhere in quantum optics. This explain the amount of papers related to many aspects of these operators and to other quantities which are related to $a$ and $a^{\dagger}$, like, for instance, the coherent states, in any of their forms, and the squeezed states.

Generalized creation and annihilation operators, $A$ and $A^{\dagger}$, have been introduced in recent years and used to construct generalized version of coherent states, see for instance [1] and references therein. However, also these new operators are usually unbounded.

In this paper we consider all these objects from the point of view of bounded operators. In other words, we propose a natural cutoff, arising from $A$ and $A^{\dagger}$ themselves, and we use this cutoff to build up new bounded operators, their free quantum evolution and what we will call the quasi-coherent states.

This paper is organized as follows: in the next section we discuss the mathematical background and we introduce the cutoff. In Section III we analyze the algebraic dynamics related to the free regularized hamiltonian using topological results connected to the so called quasi-* algebras, [5, 6, both at a finite level and at the level of the derivations. Section IV is devoted to the introduction and the analysis of quasi-coherent states, using both the Ali's and the GazeauKlauder's languages, [1, 10. We conclude the paper with a short section where some connections

with quons are discussed, [13, 9], and where it is shown that quons are just particular cases of a much more general situation.

\section{Mathematical ingredients}

Let $\mathcal{H}$ be a separable Hilbert space and $\left\{\Phi_{n}, n \in \mathbb{N}_{0}, \mathbb{N}_{0}=0,1,2 \ldots\right\}$ an o.n. basis of $\mathcal{H}$. We define the following operators

$$
P_{i, j}=\left|\Phi_{i}><\Phi_{j}\right|, \quad P_{i}=P_{i, i}, \quad Q_{L}=\sum_{i=0}^{L} P_{i}
$$


where we have used the Dirac bra-ket notation. They satisfy the following properties:

$$
\left\|P_{i, j}\right\|=1, \quad\left\|Q_{L}\right\|=1, \quad P_{i, j} P_{k, l}=\delta_{j, k} P_{i, l}, \quad P_{i}^{\dagger}=P_{i}, \quad Q_{L}^{\dagger}=Q_{L} \quad \forall i, j, k, l, L \in \mathbb{N}_{0},
$$

as well as $P_{i}^{2}=P_{i}$ and $Q_{L}^{2}=Q_{L}$. Therefore both $P_{i}$ and $Q_{L}$ are orthogonal projectors. It is clear that $P_{i}$ projects on the subspace of $\mathcal{H}$ generated by the single vector $\Phi_{i}$, while $Q_{L}$ projects on the subspace generated by $\left\{\Phi_{0}, \Phi_{1}, \ldots, \Phi_{L}\right\}$.

Let now $\left\{x_{l}, l \in \mathbb{N}_{0}\right\}$ be a generic sequence of non negative numbers, $x_{l} \geq 0$ for all $l \in \mathbb{N}_{0}$. We will assume that only $x_{0}$ could be equal to zero, while all the other coefficients are strictly positive. We put

$$
A_{L}=\sum_{l=0}^{L} \sqrt{x_{l+1}} P_{l, l+1}, \quad \text { so that } \quad A_{L}^{\dagger}=\sum_{l=0}^{L} \sqrt{x_{l+1}} P_{l+1, l}
$$

Both these operators are bounded, as far as $L<\infty$. Indeed a very easy estimate is the following: $\left\|A_{L}\right\|=\left\|A_{L}^{\dagger}\right\| \leq \sum_{l=0}^{L} \sqrt{x_{l}}$, which is a consequence of the fact that $\left\|P_{i, j}\right\|=1$ for all $i$ and $j$. However, we can do better since, for any $\Phi \in \mathcal{H}$,

$$
\begin{aligned}
\left\|A_{L} \phi\right\| & \leq \sum_{l=0}^{L} \sqrt{x_{l+1}}\left\|<\Phi_{l+1}, \phi>\Phi_{l+1}\right\|=\sum_{l=0}^{L} \sqrt{x_{l+1}}\left|<\Phi_{l+1}, \phi>\right| \\
& \leq \sqrt{\sum_{l=0}^{L} x_{l+1}} \sqrt{\sum_{l=0}^{L}\left|<\Phi_{l+1}, \phi>\right|^{2}} \leq\|\phi\| \sqrt{\sum_{l=0}^{L} x_{l+1},}
\end{aligned}
$$

which implies that, for all $L \in \mathbb{N}$,

$$
\left\|A_{L}\right\|=\left\|A_{L}^{\dagger}\right\| \leq d_{L}:=\sqrt{\sum_{l=0}^{L} x_{l+1}} .
$$

We can also derive a lower bound for $\left\|A_{L}\right\|$. For that it is enough to notice that $\left\|A_{L}\right\|=$ $\sup _{\phi \in \mathcal{H},\|\phi\|=1}\left\|A_{L} \phi\right\| \geq\left\|A_{L} \Phi_{j}\right\|$, for all values of $j \in \mathbb{N}_{0}$. Therefore $\left\|A_{L}\right\| \geq \sqrt{x_{j}}$, for all $j=0,1, \ldots, L$, which is strictly positive if $x_{0}>0$. If the sequence $\left\{x_{l}, l \in \mathbb{N}_{0}\right\}$ belongs to $l^{1}\left(\mathbb{N}_{0}\right)$, then we can conclude that

$$
\sqrt{x_{j}} \leq\left\|A_{L}\right\| \leq \sqrt{\|x\|_{1}}
$$

for all possible $j=0,1, \ldots, L$. We use $\|x\|_{1}$ to indicate the norm of $\left\{x_{l}, l \in \mathbb{N}_{0}\right\}$ in $l^{1}\left(\mathbb{N}_{0}\right)$. If this is the case, then it is trivial to check that $A_{L}$ converges in the uniform topology to a bounded 
operator. Indeed, for all $L>M$, we find $\left\|A_{L}-A_{M}\right\|^{2} \leq \sum_{l=M+1}^{L} x_{l+1}$, which goes to zero when $L, M \rightarrow \infty$. Obviously the same conclusion does not hold, in general, if $\left\{x_{l}, l \in \mathbb{N}_{0}\right\} \notin l^{1}\left(\mathbb{N}_{0}\right)$ and it surely does not hold if $\left\{x_{l}, l \in \mathbb{N}_{0}\right\}$ is not a bounded sequence. In this case, in fact, because of the lower bound $\left\|A_{L}\right\| \geq \sqrt{x_{j}}, \forall j=0,1, \ldots, L,\left\|A_{L}\right\| \rightarrow \infty$ when $L \rightarrow \infty$. This is rather common in concrete applications since, for example for the standard quantum harmonic oscillator, we just have $x_{k}=k$. Therefore, an explicit choice of $\left\{x_{l}\right\}$ must be dictated by whether we want $A_{L}$ to converge to a bounded operator or not (which is what we really have in mind since, as we have just discussed, it is what happens for an harmonic oscillator). A possible way to define $A$ and $A^{\dagger}$ in this situation is based on the fact that the o.n. basis $\left\{\Phi_{n}, n \in \mathbb{N}_{0}\right\}$ belongs to the domain of $A_{L}$ for each $L$. Introducing the sequence $\chi_{n}$, which is equal to 1 for all $n \geq 0$ and 0 otherwise, we can easily check that

$$
A_{L} \Phi_{k}=\left\{\begin{array}{ll}
0, & \text { if } k=0, \\
\chi_{L+1-k} \sqrt{x_{k}} \Phi_{k-1}, & \text { if } k>0,
\end{array} \quad \text { and } \quad A_{L}^{\dagger} \Phi_{k}=\chi_{L-k} \sqrt{x_{k+1}} \Phi_{k+1} .\right.
$$

As it is clear, they behave like creation and annihilation operators but with two significant differences: they give a non zero result only for a finite set of $k$ 's, corresponding to a finite dimensional subset of $\mathcal{H}$. The second difference is that the natural numbers $k$ are here replaced with a more general sequence of non negative numbers, $k \rightarrow x_{k}$, as in [1] and references therein.

The above formulas admit limit when $L \rightarrow \infty$, since $\lim _{L \rightarrow \infty} \chi_{L-k}=\lim _{L \rightarrow \infty} \chi_{L+1-k}=1$ for all fixed $k$. This is the way in which we introduce here the operators $A$ and $A^{\dagger}$ if $\left\{x_{l}, l \in\right.$ $\left.\mathbb{N}_{0}\right\} \notin l^{1}\left(\mathbb{N}_{0}\right)$ : we just put

$$
A \Phi_{k}=\left\{\begin{array}{ll}
0, & \text { if } k=0, \\
\sqrt{x_{k}} \Phi_{k-1}, & \text { if } k>0
\end{array} \quad \text { and } \quad A^{\dagger} \Phi_{k}=\sqrt{x_{k+1}} \Phi_{k+1} .\right.
$$

Of course, this does not imply that the definition (2.3) extends to $A=\sum_{l=0}^{\infty} \sqrt{x_{l+1}} P_{l, l+1}$, because the series does not converge, for generic $x_{j}$, in the usual topologies of bounded operators.

It is straightforward to check that the operators $A$ and $A_{L}$ are related in the following way:

$$
A_{L}=Q_{L+1} A Q_{L+1}, \quad A_{L}^{\dagger}=Q_{L+1} A^{\dagger} Q_{L+1} .
$$

The following equalities moreover hold true:

$$
A Q_{L+1}=Q_{L} A, \quad Q_{L+1} A^{\dagger}=A^{\dagger} Q_{L}, \quad A P_{l}=P_{l-1} A, \quad P_{l} A^{\dagger}=A^{\dagger} P_{l-1}
$$

whose proof, again, is left to the reader. Notice that the first equality implies in particular that

$$
A_{L}^{\dagger} A_{L}=Q_{L+1} A^{\dagger} A Q_{L+1}, \quad A_{L} A_{L}^{\dagger}=Q_{L} A A^{\dagger} Q_{L+1} .
$$


Indeed we have $A_{L}^{\dagger} A_{L}=Q_{L+1} A^{\dagger} Q_{L+1} Q_{L+1} A Q_{L+1}=Q_{L+1} A^{\dagger} Q_{L+1} A Q_{L+1}=Q_{L+1} A^{\dagger} A Q_{L+2}$ $Q_{L+1}=Q_{L+1} A^{\dagger} A Q_{L+1}$, while $A_{L} A_{L}^{\dagger}=Q_{L+1} A Q_{L+1} Q_{L+1} A^{\dagger} Q_{L+1}=Q_{L+1} A Q_{L+1} A^{\dagger} Q_{L+1}$ $=Q_{L+1} Q_{L} A A^{\dagger} Q_{L+1}=Q_{L} A A^{\dagger} Q_{L+1}$. Other relevant formulas are the following commutation rules

$$
\left[Q_{L}, A\right]=P_{L} A=A P_{L+1}, \quad\left[Q_{L}, A^{\dagger}\right]=-P_{L+1} A^{\dagger}=-A^{\dagger} P_{L},
$$

and

$$
\left[Q_{L}, A^{\dagger} A\right]=\left[Q_{L}, A A^{\dagger}\right]=0 .
$$

This list of useful formulas is completed by $\left\|P_{l} A P_{s}\right\|=\sqrt{x_{s}} \delta_{l+1, s}$ and by the following commutation rule,

$$
\left[A_{L}, A_{L}^{\dagger}\right]=\sum_{l=0}^{L} x_{l+1}\left(P_{l}-P_{l+1}\right) \Rightarrow\left[A_{L}, A_{L}^{\dagger}\right] \Phi_{k}= \begin{cases}x_{1} \Phi_{0}, & \text { if } k=0 \\ \left(\chi_{L-k} x_{k+1}-\chi_{L+1-k} x_{k}\right) \Phi_{k}, & \text { if } k>0 .\end{cases}
$$

This last equation, again, admits limit when $L \rightarrow \infty$, and the limit is

$$
\left[A, A^{\dagger}\right] \Phi_{k}= \begin{cases}x_{1} \Phi_{0}, & \text { if } k=0 \\ \left(x_{k+1}-x_{k}\right) \Phi_{k}, & \text { if } k>0\end{cases}
$$

Let us notice that, if $x_{k}=k$, i.e. for an harmonic oscillator, this formula becomes $\left[A, A^{\dagger}\right] \Phi_{k}=\Phi_{k}$ for all $k$, which implies that $\left[A, A^{\dagger}\right]=\mathcal{I}$, as it must be. In this case a rigorous meaning can be given to the otherwise in general formal expression $\left[A, A^{\dagger}\right]=x_{1} P_{0}+\sum_{l=1}^{\infty}\left(x_{l+1}-x_{l}\right) P_{l}$, which is equal, in this case, to $\left[A, A^{\dagger}\right]=\sum_{l=0}^{\infty} P_{l}=\mathcal{I}$. However, this is not the only case in which this expression is well defined: suppose indeed that the following hold: $x_{l+1} \geq x_{l}$ for all $l \in \mathbb{N}_{0}$ and $\lim _{l \rightarrow \infty} x_{l}=\bar{x}<\infty$. In this case we have

$$
\begin{gathered}
\left\|\sum_{l=1}^{\infty}\left(x_{l+1}-x_{l}\right) P_{l}\right\| \leq \sum_{l=1}^{\infty}\left(x_{l+1}-x_{l}\right)\left\|P_{l}\right\|=\sum_{l=1}^{\infty}\left(x_{l+1}-x_{l}\right)=\left(x_{2}-x_{1}\right)+\left(x_{3}-x_{2}\right)+\left(x_{4}-x_{3}\right)+\ldots= \\
=\lim _{l \rightarrow \infty} x_{l}-x_{1}=\bar{x}-x_{1} .
\end{gathered}
$$

This implies that $\left[A, A^{\dagger}\right]=x_{1} P_{0}+\sum_{l=1}^{\infty}\left(x_{l+1}-x_{l}\right) P_{l}$ is well defined in the uniform topology, even if the sequence of the $x_{j}$ 's does not converge to zero. This is not a big surprise: unbounded operators may have bounded commutators! 
In Section IV we will make use of other properties of the operators $A_{L}$ and $A_{L}^{\dagger}$. In particular using (2.3), (2.8) and (2.9), it is possible to check that

$$
\left\{\begin{array}{l}
\left(A_{L}^{\dagger}\right)^{2}=\sum_{l=0}^{L-1} \sqrt{x_{l+1} x_{l+2}} P_{l+2, l}=\left(A^{\dagger}\right)^{2} Q_{L-1}, \\
\left(A_{L}^{\dagger}\right)^{3}=\sum_{l=0}^{L-2} \sqrt{x_{l+1} x_{l+2} x_{l+3}} P_{l+3, l}=\left(A^{\dagger}\right)^{3} Q_{L-2}, \\
\ldots \ldots \ldots \\
\left(A_{L}^{\dagger}\right)^{L}=\sum_{l=0}^{1} \sqrt{x_{l+1} \ldots x_{l+L}} P_{l+L, l}=\left(A^{\dagger}\right)^{L} Q_{1}, \\
\left(A_{L}^{\dagger}\right)^{L+1}=\sqrt{x_{1} \ldots x_{L+1}} P_{L+1,0}=\left(A^{\dagger}\right)^{L+1} Q_{0}, \\
\left(A_{L}^{\dagger}\right)^{L+2}=\left(A_{L}^{\dagger}\right)^{L+3}=\ldots=0
\end{array}\right.
$$

This behavior is interesting because it extends, in a certain sense, what happens for fermions: if $b$ and $b^{\dagger}$ are fermion annihilation and creation operators, then it is well known that $b^{2}=\left(b^{\dagger}\right)^{2}=0$. In this case, $A_{L}, A_{L}^{2}, \ldots A_{L}^{L+1}$ are different from zero but all the other powers are zero, and this result does not depend on the original choice of $x_{j}$. It may be worth noticing, however, that $\left\{A_{L}, A_{L}^{\dagger}\right\}=\mathcal{I}$ is in general not satisfied, not even in an approximated form.

\section{A generalized harmonic oscillator and its algebraic dynamics}

The operators $A_{L}$ and $A_{L}^{\dagger}$ are closely related to those introduced in [5] in connection with a standard harmonic oscillator. In this section we will repeat in some details and giving more results the same analysis given in [5] for our generalized model. Of course, some differences will arise at the beginning because of the different point of view we are considering here.

First of all let us remark that the o.n. basis of $\mathcal{H},\left\{\Phi_{j}\right\}$, belongs to the domain of all the relevant operators we will consider here: $A, A^{\dagger}, A^{\dagger} A, A A^{\dagger}$, as well as their regularized counterparts, i.e. those obtained replacing the generic operator $X$ with $X_{L}:=Q_{L+1} X Q_{L+1}$. Actually, they do much more than this: they belong to the domain of all the powers of these operators. Therefore we have, for instance, $\Phi_{j} \in \mathcal{D}:=D^{\infty}\left(H_{o}\right):=\bigcap_{k \geq 0} D\left(H_{o}^{k}\right)$, for all $j$, where $H_{o}=A^{\dagger} A$. The set $\mathcal{D}$ is dense in $\mathcal{H}$. As in [5] we introduce the ${ }^{*}$-algebra $\mathcal{L}^{+}(\mathcal{D})$ of all the closable operators defined on $\mathcal{D}$ which, together with their adjoints, map $\mathcal{D}$ into itself. It is clear that all powers of $A$ and $A^{\dagger}$ belong to this set. As we have already seen, $A_{L}$ and $A_{L}^{+}$belong to $B(\mathcal{H})$, but they also belong to $\mathcal{L}^{\dagger}(\mathcal{D})$ for any $L$.

In [12], the topological structures of both $\mathcal{D}$ and $\mathcal{L}^{+}(\mathcal{D})$ are discussed in details; in particular, 
in $\mathcal{D}$ the topology is defined by the following seminorms:

$$
\phi \in \mathcal{D} \rightarrow\|\phi\|_{n} \equiv\left\|H^{n} \phi\right\|,
$$

where $n$ is a natural integer, $H$ is the closure of $H_{o}$ and \|\| is the norm of $\mathcal{H}$. The topology in $\mathcal{L}^{+}(\mathcal{D})$ is introduced in the following way. We start defining the set $\mathcal{C}$ of all the positive, bounded and continuous functions $f(x)$ on $\mathbb{R}_{+}$, which are decreasing faster than any inverse power of $x$ : $\sup _{x \in \mathbb{R}_{+}} f(x) x^{n}<\infty$ for each $n \in \mathbb{N}_{0}$. The seminorms on $\mathcal{L}^{+}(\mathcal{D})$ are labelled by functions in $\mathcal{C}$ and by the integers $\mathbb{N}_{0}$. We have

$$
X \in \mathcal{L}^{+}(\mathcal{D}) \rightarrow\|X\|^{f, k} \equiv \max \left\{\left\|f(H) X H^{k}\right\|,\left\|H^{k} X f(H)\right\|\right\}
$$

Here \|\| is the usual norm in $B(\mathcal{H})$. We use for this norm the same notation as in equation (3.1) since there is no possibility of confusion. We observe that definition (3.2) implies that $\|X\|^{f, k}=\left\|X^{\dagger}\right\|^{f, k}$ for each $X \in \mathcal{L}^{\dagger}(\mathcal{D})$, where $X^{\dagger}=\left.X^{*}\right|_{\mathcal{D}}$. We call $\tau$ the topology on $\mathcal{L}^{+}(\mathcal{D})$ defined by the seminorms (3.2). In [12] it has been proved that $\mathcal{L}^{+}(\mathcal{D})[\tau]$ is a complete locally convex topological $*$-algebra.

Notice that the two contributions in the definition (3.2) are exactly of the same form. Therefore, the estimate of $\left\|f(H) X H^{k}\right\|$ is very similar to the estimate of $\left\|H^{k} X f(H)\right\|$. This is why, from now on, we will identify $\|X\|^{f, k}$ simply with $\left\|f(H) X H^{k}\right\|$.

With this in mind, and by means of the spectral decomposition for $H, H=\sum_{l=1}^{\infty} x_{l} P_{l}$, we see that the seminorms can be estimated as follows:

$$
X \in \mathcal{L}^{+}(\mathcal{D}) \longrightarrow\|X\|^{f, k} \leq \sum_{l, s=1}^{\infty} f\left(x_{l}\right) x_{s}^{k}\left\|P_{l} X P_{s}\right\| .
$$

It is possible to check that, for all $X \in \mathcal{L}^{+}(\mathcal{D})$, the operator $X_{L-1}:=Q_{L} X Q_{L}$ belongs to $B(\mathcal{H})$. Indeed we have $\left\|X_{L-1}\right\| \leq\left\|X Q_{L}\right\|=\sup _{\varphi \in \mathcal{H}\|\varphi\|=1}\left\|X Q_{L} \varphi\right\|<\infty$ since, for each fixed $L$ and for each $\varphi \in \mathcal{H}, Q_{L} \varphi$ belongs to a finite dimensional Hilbert space. In particular we have

$$
H_{L}=Q_{L+1} H Q_{L+1}=A_{L}^{\dagger} A_{L} \in B(\mathcal{H})
$$

We can easily prove the following

Lemma 1 Suppose that the sequence $\left\{x_{s}\right\}$ is increasing to $+\infty$ when $s \rightarrow \infty$. Then, for each $l \in \mathbb{N}_{0}, \lim _{L \rightarrow \infty}\left\|H^{-l}\left(\mathcal{I}-Q_{L}\right)\right\|=0$. Moreover, for each $X \in \mathcal{L}^{\dagger}(\mathcal{D}), \tau-\lim _{L \rightarrow \infty} X_{L}=X$. 
Proof - Since $H^{-l}=\sum_{s=1}^{\infty} x_{s}^{-l} P_{s}$, and $\mathcal{I}-Q_{L}=\sum_{s=L+1}^{\infty} P_{s}$, then $H^{-l}\left(\mathcal{I}-Q_{L}\right)=\sum_{s=L+1}^{\infty} x_{s}^{-l} P_{s}$. Therefore, for each $\varphi \in \mathcal{H}$,

$$
\begin{aligned}
& \left\|H^{-l}\left(\mathcal{I}-Q_{L}\right) \varphi\right\|^{2}=\left\|H^{-l}\left(\mathcal{I}-Q_{L}\right) \varphi\right\|^{2}=<\varphi, H^{-2 l}\left(\mathcal{I}-Q_{L}\right) \varphi>= \\
& =\left(\sum_{s=L+1}^{\infty} x_{s}^{-2 l}\left\langle\varphi, P_{s} \varphi>\right) \leq \frac{1}{x_{L+1}^{2 l}} \sum_{s=0}^{\infty}<\varphi, P_{s} \varphi>\leq \frac{1}{x_{L+1}^{2 l}}\|\varphi\|^{2},\right.
\end{aligned}
$$

so that the first statement follows from of our assumption on $x_{l}$.

The proof of the second statement is very similar to that given in [6], and will not be repeated here.

Let us now define, for each $X \in \mathcal{L}^{\dagger}(\mathcal{D}), \alpha_{L}^{t}(X)=e^{i H_{L} t} X e^{-i H_{L} t}$. This operator satisfies the following, well defined, Heisenberg equation of motion $\frac{d}{d t} \alpha_{L}^{t}(X)=i\left[H_{L}, \alpha_{L}^{t}(X)\right]$. Notice that, on the contrary, the formal equation of motion $\frac{d}{d t} \alpha^{t}(X)=i\left[H, \alpha^{t}(X)\right]$ needs not to be well defined because of domain problems. However, it is easy to check that the sequence $\left\{\alpha_{L}^{t}(X)\right\}$ define the algebraic dynamics of $X$ in the following sense:

Proposition 2 Suppose that the sequence $\left\{x_{s}\right\}$ is such that $\exists n_{o} \in \mathbb{N}$ such that $\left\{x_{s}^{-n_{o}}\right\}$ belongs to $l^{1}(\mathbb{N})$. Then the sequences $\left\{e^{i H_{L} t}\right\}$ and $\left\{\alpha_{L}^{t}(X)\right\}$ are both $\tau-$ Cauchy in $\mathcal{L}^{+}(\mathcal{D})$.

Proof - Since $\left[H_{L}, H_{M}\right]=\left[H_{L}, H\right]=0$ for each $L$ and $M, M>L$, it is easy to check that

$$
I_{L M}:=\left\|f(H)\left(e^{i H_{L} t}-e^{i H_{M} t}\right) H^{k}\right\|=2\left\|f(H) \sin \left(\frac{H_{M L} t}{2}\right) H^{k}\right\|,
$$

where $H_{M L}=H_{M}-H_{L}$. Using the spectral decomposition of $H$ we further get

$$
I_{L M} \leq 2 \sum_{s=L+1}^{M} f\left(x_{s}\right) x_{s}^{k}\left|\sin \left(\frac{x_{s} t}{2}\right)\right|\left\|P_{s}\right\| \leq 2 \sum_{s=L+1}^{M} f\left(x_{s}\right) x_{s}^{k},
$$

which goes to zero when $M, L \rightarrow \infty$ for any divergent sequence $\left\{x_{n}\right\}$ satisfying our assumption since $f(x)$ belongs to $\mathcal{C}$. This is because surely there exists a positive constant $c>0$ such that $f\left(x_{s}\right) \leq \frac{c}{x_{s}^{k+n_{o}}}$ for each $s \in \mathbb{N}_{0}$, so that $I_{L M} \leq 2 c \sum_{s=L+1}^{M} \frac{1}{x_{s}^{n_{o}}}$.

To prove now that $\left\{\alpha_{L}^{t}(X)\right\}$ is $\tau$-Cauchy for each $X \in \mathcal{L}^{\dagger}(\mathcal{D})$ we consider the seminorm $G_{L M}:=\left\|f(H)\left(\alpha_{L}^{t}(X)-\alpha_{M}^{t}(X)\right) H^{k}\right\|$ which, adding and subtracting $e^{i H_{L} t} X e^{-i H_{M} t}$, can be estimated as follows

$$
G_{L M} \leq\left\|f(H) X\left(e^{-i H_{L} t}-e^{-i H_{M} t}\right) H^{k}\right\|+\left\|f(H)\left(e^{i H_{L} t}-e^{i H_{M} t}\right) X H^{k}\right\| \leq
$$




$$
\leq\left\|f(H) X H^{k+n_{o}}\right\|\left\|H^{-n_{o}}\left(e^{-i H_{L} t}-e^{-i H_{M} t}\right)\right\|+\left\|H^{-n_{o}}\left(e^{i H_{L} t}-e^{i H_{M} t}\right)\right\|\left\|H^{n_{o}} f(H) X H^{k}\right\|,
$$

where $n_{o}$, at this stage, can be an arbitrary natural number. Of course, both $\left\|f(H) X H^{k+n_{o}}\right\|$ and $\left\|H^{n_{o}} f(H) X H^{k}\right\|$ are finite, since $X \in \mathcal{L}^{\dagger}(\mathcal{D})$. We now choose the number $n_{o}$ in such a way that $\left\|H^{-n_{o}}\left(e^{ \pm i H_{L} t}-e^{ \pm i H_{M} t}\right)\right\| \rightarrow 0$ when $L, M \rightarrow \infty$. This can be easily done when the sequence $\left\{x_{s}\right\}$ is given: it is enough to take $n_{o}$ in such a way that $\left\{x_{s}^{-n_{o}}\right\} \in l^{1}(\mathbb{N}), \sum_{s=1}^{\infty} x_{s}^{-n_{o}}<\infty$. Indeed, with this choice and with the same estimates as above, we get for instance

$$
\left\|H^{-n_{o}}\left(e^{-i H_{L} t}-e^{-i H_{M} t}\right)\right\|=2\left\|\sum_{s=L+1}^{M} x_{s}^{-n_{o}} \sin \left(\frac{x_{s} t}{2}\right) P_{s}\right\| \leq \sum_{s=L+1}^{M} x_{s}^{-n_{o}} \rightarrow 0,
$$

when $M, L \rightarrow \infty$.

This Proposition can be used to define both $e^{ \pm i H t}$ and $\alpha^{t}(X)$ for each $X \in \mathcal{L}^{\dagger}(\mathcal{D}): e^{ \pm i H t}:=$ $\tau-\lim _{L} e^{ \pm i H_{L} t}$ and $\alpha^{t}(X):=\tau-\lim _{L} e^{i H_{L} t} X e^{-i H_{L} t}$, making no use of intrinsically ill-defined series expansions for the unbounded operator $H$. Of course, one could also try to give a meaning to these quantities using the spectral theorem. However, the procedure presented here seems promising for a possible extension to the situation in which no hamiltonian operator exists, as for instance for mean field models, which are crucial in concrete physical applications. A deeper analysis on this aspect is in progress.

Another interesting remark concerns the following formula, which relates $e^{i H_{L} t}$ and $e^{i H t}$, which is obtained under the assumption that all the quantities are well defined:

$$
e^{i H_{L} t}=\mathcal{I}-Q_{L+1}+Q_{L+1} e^{i H t} Q_{L+1}
$$

Indeed, since all the powers of $H$ belong to $\mathcal{L}^{+}(\mathcal{D})$, while $H_{L}^{n}$ belongs to $B(\mathcal{H})$ for each $L$ and each $n$, it is straightforward to check that $H_{L}^{n}=Q_{L+1} H^{n} Q_{L+1}$. Therefore

$$
\begin{gathered}
e^{i H_{L} t}=\mathcal{I}+i H_{L} t+\frac{1}{2 !}\left(i H_{L} t\right)^{2}+\frac{1}{3 !}\left(i H_{L} t\right)^{3}+\ldots= \\
=\mathcal{I}-Q_{L+1}+Q_{L+1}\left(\mathcal{I}+i H t+\frac{1}{2 !}(i H t)^{2}+\frac{1}{3 !}(i H t)^{3}+\ldots\right) Q_{L+1},
\end{gathered}
$$

which returns (3.5) if the series above converges. In general, therefore, (3.5) must be seen only as a formal equality.

Let us now consider the dynamics at the infinitesimal level, that is at the level of the derivations. This kind of problems has been recently analyzed in [7] and [8]. The first obvious remark 
is that, since $\tau-\lim _{L} H_{L}^{k}=H^{k}$ for all $k \in \mathbb{N}_{o}$, and since the multiplication is separately continuous in the topology $\tau$, the regularized derivation $\delta_{L}(X)=i\left[H_{L}, X\right]$ converges to the derivation $\delta(X)=i[H, X]$ in $\tau$, for each operator $X \in \mathcal{L}^{\dagger}(\mathcal{D})$. However, this is not enough to conclude that also the repeated commutators do converge, since these contain contributions like $H_{L}^{n} X H_{L}^{m}$. Nevertheless, introducing recursively the $k-t h$ regularized derivation as $\delta_{L}^{k}(X)=i\left[H_{L}, \delta_{L}^{k-1}(X)\right]$, the following Proposition holds true:

Proposition 3 For each $k \geq 1$ and for each $X \in \mathcal{L}^{\dagger}(\mathcal{D}), \tau-\lim _{L} \delta_{L}^{k}(X)$ exists in $\mathcal{L}^{\dagger}(\mathcal{D})$, and it defines an operator which we call $\delta^{(k)}(X)$, and which obeys the following recursion formula: $\delta^{(k)}(X)=i\left[H, \delta^{(k-1)}(X)\right]$.

Proof - Our claim is proved by induction. The statement is clearly true for $k=1$. Suppose therefore it holds for a fixed $k$, that is that $\tau-\lim _{L} \delta_{L}^{k}(X)=\delta^{(k)}(X) \in \mathcal{L}^{\dagger}(\mathcal{D})$. This means that $\delta^{(k+1)}(X)=i\left[H, \delta^{(k)}(X)\right]$ is well defined in $\mathcal{L}^{\dagger}(\mathcal{D})$. Then we have

$$
\begin{gathered}
\left\|f(H)\left(\delta_{L}^{k+1}(X)-\delta^{(k+1)}(X)\right) H^{l}\right\| \leq \\
\leq\left\|f(H)\left(H_{L} \delta_{L}^{k}(X)-H \delta^{(k)}(X)\right) H^{l}\right\|+\left\|f(H)\left(\delta_{L}^{k}(X) H_{L}-\delta^{(k)}(X) H\right) H^{l}\right\|=: P_{1}^{L}+P_{2}^{L},
\end{gathered}
$$

with obvious notation. Adding and subtracting $H \delta_{L}^{k}(X)$ in $P_{1}^{L}$ we get

$$
P_{1}^{L} \leq\left\|f(H)\left(H_{L}-H\right) \delta_{L}^{k}(X) H^{l}\right\|+\left\|f(H) H\left(\delta_{L}^{(k)}(X)-\delta^{(k)}(X)\right) H^{l}\right\|=: P_{1, A}^{L}+P_{1, B}^{L}
$$

again with obvious notation. Since $x f(x) \in \mathcal{C}$ for each $f(x) \in \mathcal{C}$, our induction assumption easily implies that $\lim _{L} P_{1, B}^{L}=0$. As for $P_{1, A}^{L}$, recalling that $\left[H, H_{L}\right]=\left[H, Q_{L}\right]=0$, we deduce that

$$
P_{1, A}^{L} \leq\left\|H^{-1}\left(Q_{L}-\mathcal{I}\right)\right\|\left\|f(H) H^{2} \delta_{L}^{k}(X) H^{l}\right\| \rightarrow 0
$$

when $L \rightarrow \infty$ using Lemma 1 above, since $x^{2} f(x) \in \mathcal{C}$ and since $\delta_{L}^{k}(X)$, being $\tau$-convergent, is necessarily $\tau$-bounded. Therefore we have $\lim _{L} P_{1}^{L}=0$. The proof of $\lim _{L} P_{2}^{L}=0$ follows essentially the same steps.

It is worth remarking that we have used here, as in [6], the notation $\delta^{(k)}(X)$ instead of a maybe more natural $\delta^{k}(X)$ since this cannot in general be defined, as one expects, as $\delta^{k}(X)=$ $i\left[H, \delta^{k-1}(X)\right]$, because the rhs of this equation is not everywhere defined when $H$ is unbounded. 
What this Proposition incidentally proves is that the series in $\alpha_{L}^{t}(X)=\sum_{k=0}^{\infty} \frac{t^{k}}{k !} \delta_{L}^{k}(X)$ is term to term $\tau$-convergent to $\sum_{k=0}^{\infty} \frac{t^{k}}{k !} \delta^{(k)}(X)$, which could be used to define $\alpha^{t}(X)$. Using our previous results we can therefore state the following: for each $X \in \mathcal{L}^{\dagger}(\mathcal{D})$ and for each $t \in \mathbb{R}$,

$$
e^{i H t} X e^{-i H t}=\alpha^{t}(X)=\tau-\lim _{L \rightarrow \infty} \alpha_{L}^{t}(X)=\tau-\lim _{L \rightarrow \infty} \sum_{k=0}^{\infty} \frac{t^{k}}{k !} \delta_{L}^{k}(X)
$$

\section{Approximated coherent states}

This section is devoted to the possibility of using the operators $A_{L}$ and $A_{L}^{\dagger}$ to generate approximate coherent states (ACS), that is vectors, depending on the regularizing parameter $L$, which share all the properties of the coherent states in the limit $L \rightarrow \infty$ and an approximated version of these if $L$ is left finite.

In the old literature, see [11] for instance, a standard coherent state (SCS) is a vector arising from the action of the unitary operator $U(z)=e^{z a^{\dagger}-\bar{z} a}, z \in \mathbb{C}$ and $\left[a, a^{\dagger}\right]=\mathcal{I}$, on the vacuum of $a, \Phi_{0}, a \Phi_{0}=0: \mid z>=U(z) \Phi_{0}$. These normalized vectors can be written in other equivalent ways, introducing the o.n. basis $\left\{\Phi_{n}, n \in \mathbb{N}_{0}\right\}$ where $\Phi_{n}=\frac{\left(a^{\dagger}\right)^{n}}{\sqrt{n !}} \Phi_{0}$, as follows:

$$
\mid z>=U(z) \Phi_{0}=e^{-|z|^{2} / 2} e^{z a^{\dagger}} \Phi_{0}=e^{-|z|^{2} / 2} \sum_{k=0}^{\infty} \frac{z^{n}}{\sqrt{n !}} \Phi_{n} .
$$

They share a lot of interesting properties, among which the most interesting for us are the following:

1. $\mid z>$ is an eigenstate of $a: a|z>=z| z>$.

2. They satisfy a resolution of the identity: $\frac{1}{\pi} \int d^{2} z|z><z|=\mathcal{I}$.

3. They saturate the Heisenberg uncertainty principle: let $q=\frac{a+a^{\dagger}}{\sqrt{2}}, p=\frac{a-a^{\dagger}}{i \sqrt{2}},(\Delta X)^{2}=<$ $X^{2}>-<X>^{2}$ for $X=q, p$, then $\Delta q \Delta p=\frac{1}{2}$.

These properties are recovered using a different definition for the coherent states, see [1] and references therein, definition which generalizes the one above and which appears strictly related to the procedure introduced in Section II. Starting from a sequence $\left\{x_{l}, l \in \mathbb{N}_{0}\right\}$, of non negative numbers, $x_{l} \geq 0$ for all $l \in \mathbb{N}_{0}$, it is possible to define some vectors, parametrized by a complex $z$, as follows:

$$
\Xi(z):=N\left(|z|^{2}\right)^{-1 / 2} \sum_{k=0}^{\infty} \frac{z^{n}}{\sqrt{x_{n} !}} \Phi_{n}
$$


where $N\left(|z|^{2}\right)=\sum_{k=0}^{\infty} \frac{|z|^{2 n}}{x_{n} !}$, so that $<\Xi, \Xi>=1$ for all $|z| \leq \rho, \rho$ being the radius of convergence of the series for $N$, and where $x_{0} !=1$ and $x_{n} !=x_{1} x_{2} \ldots x_{n}$. In particular, if $A$ is the operator in (2.7), we have $A \Xi(z)=z \Xi(z)$, so that these generalized coherent states (GCS) are again eigenstates of the generalized annihilation operator $A$. Moreover, in [1] it is also shown that the existence of a resolution of the identity, that is the existence of a measure $d \nu(z, \bar{z})$ such that $\int_{C_{\rho}} N\left(|z|^{2}\right)|\Xi(z)><\Xi(z)| d \nu(z, \bar{z})=\mathcal{I}$, is related to the existence of a solution of the following moment problem: we put $z=r e^{i \theta}, d \nu(z, \bar{z})=d \theta d \lambda(r), C_{\rho}=\left\{z=r e^{i \theta}, \theta \in[0,2 \pi[, r \in[0, \rho[\}\right.$, then we want $d \lambda(r)$ to be such that

$$
\int_{0}^{\rho} d \lambda(r) r^{2 k}=\frac{x_{k} !}{2 \pi}, \quad \forall k \in \mathbb{N}_{0}
$$

It is known that this problem has not always solution, but when it does, then a resolution of the identity can be established.

Finally, if we introduce two self-adjoint operators (the generalized position and momentum operators) $Q=\frac{A+A^{\dagger}}{\sqrt{2}}, P=\frac{A-A^{\dagger}}{i \sqrt{2}}$, then $\Xi(z)$ saturates again the Heisenberg uncertainty principle, which now can be written as

$$
\Delta Q \Delta P=\left.\frac{1}{2}\left|<A A^{\dagger}>-\right| z\right|^{2} \mid .
$$

Notice that, if $x_{k}=k$, and therefore $A=a$, this returns the standard expression: $\Delta Q \Delta P=\frac{1}{2}$.

As we observe, the three possible equivalent definitions for the SCS are replaced by an unique definition for the GCS. This is due to the fact that, since in general $\left[A, A^{\dagger}\right] \neq \mathcal{I}$, the equality $e^{z a^{\dagger}-\bar{z} a}=e^{-|z|^{2} / 2} e^{z a^{\dagger}} e^{-\bar{z} a}$ does not extend any-longer: $e^{z A^{\dagger}-\bar{z} A} \neq e^{-|z|^{2} / 2} e^{z A^{\dagger}} e^{-\bar{z} A}$. More generalizations can be found in [4], but they will have no role along this paper. Of course, since $e^{-|z|^{2} / 2} \sum_{k=0}^{\infty} \frac{z^{n}}{\sqrt{n !}} \Phi_{n}$ does not explicitly refer to the creation and annihilation operators, this can be naturally generalized as in (4.2). We want now to introduce our ACS, which should be defined starting from $A_{L}^{\sharp}=Q_{L+1} A^{\sharp} Q_{L+1}$, and we discuss their relations with GCS.

For that we define, starting from the fixed sequence $\left\{x_{l}, l \in \mathbb{N}_{0}\right\}$, the analytic function $F(z)=\sum_{k=0}^{\infty} \frac{z^{n}}{x_{n}}$ ! whose radius of convergence is exactly $\rho$. We use $F(z)$ to define our ACS in the following way:

$$
\Psi_{L}(z):=N_{\Psi_{L}}\left(|z|^{2}\right)^{-1 / 2} F\left(z A_{L}^{\dagger}\right) \Phi_{0}
$$

where $N_{\Psi_{L}}\left(|z|^{2}\right)$ must be chosen in such a way that $<\Psi_{L}(z), \Psi_{L}(z)>=1$ for all $L$ and for each $z$ inside the domain of convergence. 
If, for instance, $x_{n}=n$, then $F(z)=e^{z}$. In this case we claim that $\lim _{L \rightarrow \infty} \Psi_{L}(z)=\mid z>$, that is we recover the SCS. This statement will be proved in the following. Here we want to prove that $\Psi_{L}(z)$ can be written in another form, which is more closely related to definition (4.2) and which is useful for further considerations. The starting point for this is the result in (2.15) which states, in particular, that $\left(A_{L}^{\dagger}\right)^{j}=0$ for $j=L+2, L+3, \ldots$, and that $A_{L}^{\dagger} \Phi_{0}=\sqrt{x_{1}} \Phi_{1}$, $\left(A_{L}^{\dagger}\right)^{2} \Phi_{0}=\sqrt{x_{1} x_{2}} \Phi_{2}, \ldots,\left(A_{L}^{\dagger}\right)^{L+1} \Phi_{0}=\sqrt{x_{1} \ldots x_{L+1}} \Phi_{L+1}$. Therefore we have

$$
\begin{gathered}
\Psi_{L}(z):=N_{\Psi_{L}}\left(|z|^{2}\right)^{-1 / 2} F\left(z A_{L}^{\dagger}\right) \Phi_{0}=N_{\Psi_{L}}\left(|z|^{2}\right)^{-1 / 2} \sum_{k=0}^{\infty} \frac{\left(z A_{L}^{\dagger}\right)^{k}}{x_{k} !} \Phi_{0}= \\
=N_{\Psi_{L}}\left(|z|^{2}\right)^{-1 / 2} \sum_{k=0}^{L+1} \frac{\left(z A_{L}^{\dagger}\right)^{k}}{x_{k} !} \Phi_{0},
\end{gathered}
$$

which gives the following alternative expression for $\Psi_{L}(z)$,

$$
\Psi_{L}(z):=N_{\Psi_{L}}\left(|z|^{2}\right)^{-1 / 2} \sum_{k=0}^{L+1} \frac{z^{k}}{\sqrt{x_{k} !}} \Phi_{k} .
$$

This formula shows that $\Psi_{L}(z)$ is a finite linear combination of the $\Phi_{j}$ 's, and, recalling (4.2), is also the most natural one: it can be obtained from (4.2) simply restricting the series to the first $L+1$ terms and, as a consequence, modifying also the normalization, which must be chosen as $N_{\Psi_{L}}\left(|z|^{2}\right)=\sum_{k=0}^{L+1} \frac{|z|^{2 k}}{x_{k} !}$. Recalling definitions (2.1) and (4.2) we can easily relate $\Psi_{L}(z)$ with $\Xi(z)$ :

$$
\Psi_{L}(z)=\sqrt{\frac{N\left(|z|^{2}\right)}{N_{\Psi_{L}}\left(|z|^{2}\right)}} Q_{L+1} \Xi(z),
$$

which, of course, indicates that $\lim _{L \rightarrow \infty} \Psi_{L}(z)=\Xi(z)$, as expected.

Formulas (4.5) and (4.6) are, in a certain sense, our counterparts of (4.1) for these ACS: they extend the second and the third possibilities in (4.1) to the case in which $x_{n}$ is generic. Notice that, if $x_{n}=n$, then we recover a sort of natural cutoff of the SCS.

The ACS $\Psi_{L}(z)$ turns out to be an approximated eigenstate of $A_{L}$. Indeed we have

$$
A_{L} \Psi_{L}(z)=z \sqrt{\frac{N_{\Psi_{L-1}}\left(|z|^{2}\right)}{N_{\Psi_{L}}\left(|z|^{2}\right)}} \Psi_{L-1}(z),
$$


which formally converges, when $L \rightarrow \infty$ to the eigenvalue equation $A \Xi(z)=z \Xi(z)$. Let us first prove equation (4.8), and then we will discuss in more details what happens in the limit $L \rightarrow \infty$.

We start remarking that, for each fixed $L, A_{L} \Phi_{0}=0, A_{L} \Phi_{k}=\sqrt{x_{k}} \Phi_{k-1}$, for $k=1,2, \ldots, L+$ 1 , and $A_{L} \Phi_{k}=0$ for $k \geq L+2$. Therefore we have

$$
A_{L} \Psi_{L}(z)=N_{\Psi_{L}}\left(|z|^{2}\right)^{-1 / 2} \sum_{k=0}^{L+1} \frac{z^{k}}{\sqrt{x_{k} !}} A_{L} \Phi_{k}=N_{\Psi_{L}}\left(|z|^{2}\right)^{-1 / 2} \sum_{k=1}^{L+1} \frac{z^{k}}{\sqrt{x_{k} !}} \sqrt{x_{k}} \Phi_{k-1},
$$

so that formula (4.8) immediately follows.

As for the limit for $L$ divergent, it is possible to check that

$$
\lim _{L \rightarrow \infty}\left\|A_{L} \Psi_{L}(z)-A \Xi(z)\right\|=\lim _{L \rightarrow \infty}\left\|z \sqrt{\frac{N_{\Psi_{L-1}}\left(|z|^{2}\right)}{N_{\Psi_{L}}\left(|z|^{2}\right)}} \Psi_{L-1}(z)-z \Xi(z)\right\|=0 .
$$

We leave the proof of this statement to the reader. Here we just notice that this shows that (4.8) is an approximated version of the eigenvalue equation $A \Xi(z)=z \Xi(z)$.

We can also check that the states $\Psi_{L}(z)$ generate an approximated decomposition of the identity. Indeed, if we take $d \nu(z, \bar{z})$ as before, with $d \lambda(r)$ satisfying the moment problem in (4.3), with exactly the same strategy we can check that $\int_{C_{\rho}} N_{\Psi_{L}}\left(|z|^{2}\right)\left|\Psi_{L}(z)><\Psi_{L}(z)\right| d \nu(z, \bar{z})=$ $\sum_{k=0}^{L+1} P_{k}=Q_{L+1}$, which is not $\mathcal{I}$ but tends to $\mathcal{I}$ when $L \rightarrow \infty$ in the strong topology.

The final step of this analysis is the uncertainty relation. Again, we expect that this is saturated only in an approximated version and this is indeed what happens. Defining as usual $Q_{L}=\frac{A_{L}+A_{L}^{\dagger}}{\sqrt{2}}$ and $P_{L}=\frac{A_{L}-A_{L}^{\dagger}}{i \sqrt{2}}$, and repeating the usual computations, we find that

$$
\Delta Q_{L} \Delta P_{l}=\frac{1}{2} \sqrt{\Gamma_{2, L}^{2}-\Gamma_{1, L}^{2}}
$$

where

$$
\Gamma_{1, L}=<A_{L} A_{L}^{\dagger}>+|z|^{2} \frac{N_{\Psi_{L-2}}\left(|z|^{2}\right)}{N_{\Psi_{L-1}}\left(|z|^{2}\right)}\left(1-2 \frac{N_{\Psi_{L-2}}\left(|z|^{2}\right)}{N_{\Psi_{L-1}}\left(|z|^{2}\right)}\right)
$$

and

$$
\Gamma_{2, L}=\left(z^{2}+\bar{z}^{2}\right) \frac{1}{N_{\Psi_{L-1}}\left(|z|^{2}\right)}\left(N_{\Psi_{L-1}}\left(|z|^{2}\right)-\frac{N_{\Psi_{L-2}}\left(|z|^{2}\right)}{N_{\Psi_{L-1}}\left(|z|^{2}\right)}\right) .
$$

To check whether the Heisenberg inequality is saturated, that is if $\Delta Q_{L} \Delta P_{l}=\frac{1}{2}\left(<\left[A_{L}, A_{L}^{\dagger}\right]>\right)$, we should compute the rhs, which gives $\frac{1}{2}\left(<A_{L} A_{L}^{\dagger}>-|z|^{2} \frac{N_{\Psi_{L-2}}\left(|z|^{2}\right)}{N_{\Psi_{L-1}}\left(|z|^{2}\right)}\right)$. We see that, for $L<\infty$, in general $\Delta Q_{L} \Delta P_{l}=\frac{1}{2} \sqrt{\Gamma_{2, L}^{2}-\Gamma_{1, L}^{2}} \geq \frac{1}{2}\left(<A_{L} A_{L}^{\dagger}>-|z|^{2} \frac{N_{\Psi_{L-2}}\left(|z|^{2}\right)}{N_{\Psi_{L-1}}\left(|z|^{2}\right)}\right)$. However, 
since $\Gamma_{1, L} \rightarrow<A A^{\dagger}>-|z|^{2}$ and $\Gamma_{2, L} \rightarrow 0$, when $L \rightarrow \infty$, then $\left.\Delta Q_{L} \Delta P_{l} \rightarrow \frac{1}{2}\left|<A A^{\dagger}>-\right| z\right|^{2} \mid$ which coincides with the limit of $\frac{1}{2}\left(<A_{L} A_{L}^{\dagger}>-|z|^{2} \frac{N_{\Psi_{L-2}}\left(|z|^{2}\right)}{N_{\Psi_{L-1}}\left(|z|^{2}\right)}\right)$ for $L$ diverging. In other words, $\Psi_{L}(z)$ saturates the Heisenberg uncertainty relation in the limit $L \rightarrow \infty$.

We want now to find the explicit expression of a vector in $\mathcal{H}$ in order that it is an eigenstate of $A_{L}$. This is a sort of inverse problem of the one considered so far.

Since $\Phi_{j}$ is an o.n. basis of $\mathcal{H}$, if such an eigenstate $\Upsilon_{L}(z)$ exists it must admit an expansion like $\Upsilon_{L}(z)=\sum_{k=0}^{\infty} b_{k}^{(L)}(z) \Phi_{k}$, and the problem consists in finding the coefficients $b_{k}^{(L)}(z)$ in such a way that $A_{L} \Upsilon_{L}(z)=z \Upsilon_{L}(z)$ is satisfied. However, it is not difficult to check that this requirement implies that $b_{j}^{(L)}(z)=0$ for $j=0,1,2, \ldots$, so that no nontrivial solution of the eigenvalue equation can exist. For this reason we weaken the requirement, following a suggestion already contained in formula (4.8): we look for an approximate eigenstate of $A_{L}$, that is a vector of $\mathcal{H}$ such that $A_{L} \Upsilon_{L}(z)=z \Upsilon_{L-1}(z)$. It is convenient to expand $\Upsilon_{L}(z)$ as it follows

$$
\Upsilon_{L}(z)=\sum_{k=0}^{\infty} b_{k}^{(L+1)}(z) \Phi_{k} .
$$

We need to compute the coefficients of this expansion. In order to satisfy the equality $A_{L} \Upsilon(z)_{L}=$ $z \Upsilon(z)_{L-1}$ these must obey the following recursion formula:

$$
b_{j}^{(L+1)}(z) \sqrt{x_{j}}=z b_{j-1}^{(L)}(z)
$$

with $b_{j}^{(L+1)}(z)=0$ for each $j>L+1$. This implies that $\Upsilon_{L}(z)$ must be of the following form

$$
\Upsilon_{L}(z)=\sum_{k=0}^{L+1} b_{0}^{(L+1-k)}(z) \frac{z^{k}}{\sqrt{x_{k} !}} \Phi_{k}
$$

for any choice of $b_{0}^{(j)}(z)$, which however should be conveniently chosen if we also want $\Upsilon_{L}(z)$ to be normalized.

If we take for instance $b_{0}^{(0)}(z)=b_{0}^{(1)}(z)=\ldots=b_{0}^{(L+1)}(z)=b(z)$, then we get $\Upsilon_{L}(z)=$ $b(z) \sum_{k=0}^{L+1} \frac{z^{k}}{\sqrt{x_{k}} !} \Phi_{k}$, which coincides with (4.6) but for the normalization constant, which can be computed easily, recovering exactly the result in (4.6).

Another interesting choice of $b_{0}^{(j)}(z)$ is the following: $b_{0}^{(j)}(z)=\frac{z^{j}}{\sqrt{x_{j} !}}$, which produces the following ACS: $\Upsilon_{L}(z)=z^{L+1} \sum_{k=0}^{L+1} \frac{1}{\sqrt{x_{L+1-k} ! x_{k} !}} \Phi_{k}$. Here, as it is evident, $z$ appears only to the single power $L+1$. Notice that, again, $\Upsilon_{L}(z)$ is not normalized. In general, normalizing 
such a $\Upsilon_{L}(z)$ produce a different function $\hat{\Upsilon}_{L}(z)=N_{L}^{-1 / 2}(z) \Upsilon_{L}(z)$, which satisfies $A_{L} \hat{\Upsilon}_{L}(z)=$ $z \sqrt{\frac{N_{L-1}(z)}{N_{L}(z)}} \hat{\Upsilon}_{L-1}(z)$. We see that this does not differ significantly from $A_{L} \hat{\Upsilon}_{L}(z)=z \hat{\Upsilon}_{L-1}(z)$ for large $L$, since $N_{L-1}(z) \simeq N_{L}(z)$ in this case.

Another possible choice that we have considered is $b_{0}^{(j)}(z)=\sqrt{\frac{x_{j} !}{j !}}$. It is not hard to imagine more choices, some of which could be useful in concrete applications. In general the examples already considered show that different choices of $b_{0}^{(j)}$ produce ACS with different analytical and, possibly, physical characteristics.

Of course, the choice of the coefficients produces, in turns, the related moment problem (4.3) which must be satisfied in order to get an approximated resolution of the identity.

Remark: it is well known that coherent states are deeply connected with squeezed states, so that one could try to repeat the same analysis considered here for these states. This will be discussed in a forthcoming paper.

We end this section considering a different kind of coherent states, those first introduced by Gazeau and Klauder, [10], and generalized in [3]. These states, labelled by two real numbers $J$ and $\gamma$, can be written in terms of the o.n. basis of a self-adjoint operator $H=H^{\dagger},|n\rangle$, as

$$
\left|J, \gamma>=N(J)^{-1} \sum_{n=0}^{\infty} \frac{J^{n / 2} e^{-i \epsilon_{n} \gamma}}{\sqrt{\rho_{n}}}\right| n>,
$$

where $N(J)^{2}=\sum_{n=0}^{\infty} \frac{J^{n}}{\rho_{n}}, H\left|n>=\omega \epsilon_{n}\right| n>$, with $0=\epsilon_{0}<\epsilon_{1}<\epsilon_{2}<\ldots$ It may be worth noticing that the normalization of these GK-states is $N(J)^{-1}$ and formally differs from the one used by Ali et al, [1]. We adopt here the same notation as in the original papers. These states satisfy the following properties:

1. if there exists a non negative function, $\rho(u)$, such that $\int_{0}^{R} \rho(u) u^{n} d u=\rho_{n}$ for all $n \geq 0$, where $R$ is the radius of convergence of $N(J)$, then, introducing a measure $d \nu(J, \gamma)=$ $N(J)^{2} \rho(J) d J d \nu(\gamma)$, with $\int_{\mathbb{R}} \ldots d \nu(\gamma)=\lim _{\Gamma \rightarrow \infty} \frac{1}{2 \Gamma} \int_{-\Gamma}^{\Gamma} \ldots d \gamma$, the following resolution of the identity is satisfied:

$$
\int_{C_{R}} d \nu(J, \gamma)|J, \gamma><J, \gamma|=\int_{0}^{R} N(J)^{2} \rho(J) d J \int_{\mathbb{R}} d \nu(\gamma)|J, \gamma><J, \gamma|=\mathcal{I}
$$

2. the states $\mid J, \gamma>$ are temporarily stable:

$$
e^{-i H t}|J, \gamma>=| J, \gamma+\omega t>, \quad \forall t \in \mathbb{R} ;
$$


3. if $\rho_{n}=x_{n}$ ! then they satisfy the action identity:

$$
<J, \gamma|H| J, \gamma>=J \omega
$$

4. they are continuous: if $(J, \gamma) \rightarrow\left(J_{0}, \gamma_{0}\right)$ then $\left\||J, \gamma>-| J_{0}, \gamma_{0}>\right\| \rightarrow 0$.

It is interesting to observe that the states $\mid J, \gamma>$ are eigenstates of the following $\gamma$ - depending annihilation-like operator $a_{\gamma}$ defined on $\mid n>$ as follows:

$$
a_{\gamma} \mid n>= \begin{cases}0, & \text { if } n=0 \\ \sqrt{\epsilon_{n}} e^{i\left(\epsilon_{n}-\epsilon_{n-1}\right) \gamma} \mid n-1>, & \text { if } n>0\end{cases}
$$

whose adjoint acts as $a_{\gamma}^{\dagger}\left|n>=\sqrt{\epsilon_{n+1}} e^{-i\left(\epsilon_{n+1}-\epsilon_{n}\right) \gamma}\right| n+1>$. This shows that $H$ can be written as $H=\omega a_{\gamma}^{\dagger} a_{\gamma}$. With standard computations we can also check that

$$
a_{\gamma}|J, \gamma>=\sqrt{J}| J, \gamma>\text {. }
$$

However, it should be stressed that $\mid J, \gamma>$ is not an eigenstate of $a_{\gamma^{\prime}}$ if $\gamma \neq \gamma^{\prime}$.

Using the suggestion coming from formula (4.7) we define new vectors $\mid J, \gamma ; L>$ as

$$
\left|J, \gamma ; L>=\frac{N(J)}{N_{L}(J)} Q_{L+1}\right| J, \gamma>
$$

where as usual $Q_{L+1}=\sum_{k=0}^{L+1}|k><k|$ and where $N_{L}(J)^{2}=\sum_{k=0}^{L+1} \frac{J^{n}}{\rho_{n}}$. These states are interesting, since they satisfy the following properties:

1. they can be written as

$$
\left|J, \gamma ; L>=\frac{1}{N_{L}(J)} \sum_{n=0}^{L+1} \frac{J^{n / 2} e^{-i \epsilon_{n} \gamma}}{\sqrt{\rho_{n}}}\right| n>,
$$

2. if there exists a function, $\rho(u)$ with the same features as above, then, introducing a measure $d \nu_{L}(J, \gamma)=N_{L}(J)^{2} \rho(J) d J d \nu(\gamma)$, with $d \nu(\gamma)$ as before, the following identity holds true:

$$
\int_{C_{R}} d \nu_{L}(J, \gamma)|J, \gamma ; L><J, \gamma ; L|=Q_{L+1}
$$

3. the states $\mid J, \gamma ; L>$ are temporarily stable:

$$
e^{-i H t}|J, \gamma ; L>=| J, \gamma+\omega t ; L>\quad \forall t \in \mathbb{R}, \forall L ;
$$


4. they satisfy the following identity:

$$
<J, \gamma ; L\left|H_{L}\right| J, \gamma ; L>=J \omega\left(\frac{N_{L-1}(J)}{N_{L}(J)}\right)^{2}
$$

5. they are continuous: if $(J, \gamma) \rightarrow\left(J_{0}, \gamma_{0}\right)$ then $\left\||J, \gamma ; L>-| J_{0}, \gamma_{0} ; L>\right\| \rightarrow 0$.

The proofs of these statements are not significantly different from that of the original ones and will be omitted here. As we see, the states $\mid J, \gamma ; L>$ satisfy, mostly in an approximated version, the requirement of the GK-coherent states. A relevant feature is that they satisfy temporal stability exactly for each value of $L$, even if they differ from the original GK-states since they are just a finite linear combinations of the eigenstates $\mid n>$ of $H$.

\section{Connections with quons}

In a series of papers [13, 9] many people have introduced and analyzed a different kind of elementary particles, the so called quons. They interpolate between bosons and fermions, in the sense that they satisfy a modified version of the canonical commutation relations which depend on a parameter $q$. More explicitly, when $q=1$ these particles obey CCR while, if $q=-1$, they obey CAR. Different kind of quons have been proposed in the literature but maybe the most common are those satisfying the following q-mutators:

$$
a a^{\dagger}-q a^{\dagger} a=\mathcal{I} \quad \text { or } \quad a a^{\dagger}-q a^{\dagger} a=q^{-2 \hat{N}} \mathcal{I}
$$

where $\hat{N}$ is the number operator: $\hat{N} \Phi_{j}=j \Phi_{j}$, [13]. We will call respectively first and second kind quons those satisfying the first or the second q-mutator above. In this section we will discuss briefly the relation between these quons, and other obeying other generalized q-mutation relations, with the operators $A$ and $A^{\dagger}$ introduced in Section II, extending some results first discussed by Ali et al., [2].

The spectral decompositions for the operators $A^{\dagger} A$ and $A A^{\dagger}$ are respectively $\sum_{l=0}^{\infty} x_{l+1} P_{l+1}$ and $\sum_{l=0}^{\infty} x_{l+1} P_{l}$, which implies that

$$
A A^{\dagger}-q A^{\dagger} A=x_{1} P_{0}+\left(x_{2}-q x_{1}\right) P_{1}+\left(x_{3}-q x_{2}\right) P_{2}+\ldots
$$

This expansion is equal to the identity operator $\mathcal{I}=\sum_{l=0}^{\infty} P_{l}$ if and only if $x_{1}=1, x_{2}-q x_{1}=$ $x_{3}-q x_{2}=x_{4}-q x_{3}=\ldots=1$. Therefore we find

$$
x_{n+1}=1+q+q^{2}+\ldots+q^{n}= \begin{cases}n+1, & \text { if } q=1 \\ \frac{1-q^{n+1}}{1-q}, & \text { if } q \neq 1\end{cases}
$$


It is worth noticing that the result $x_{n}=n$ when $q=1$ is expected: in this case, indeed, the q-mutator rules in (5.1), as well as the ones we will consider below, return the CCR. Let us also remind that $H_{o} \Phi_{n}=A^{\dagger} A \Phi_{n}=x_{n} \Phi_{n}$. This means that, if we are interested in using the results obtained in the previous sections to the analysis of first kind quons, then the sequence $\left\{x_{j}\right\}$ must be chosen as in (5.2). We notice also that this equation, if $q \notin[-1,1[$, produce unbounded operators. In other words, we could use the results given in Section III and IV to study the algebraic dynamics of free quons and the ACS associated to them.

A standard problem in this topic is related to the definition of the number operator $\hat{N}$. For first kind quons this operator satisfies the equation $H_{O}=\frac{1-q^{\hat{N}}}{1-q}$, [13], and can be written as $\hat{N}=\frac{1}{\log (q)} \log \left(\mathcal{I}-H_{o}(1-q)\right)$.

The same analysis can be carried out for the second kind quons. In this case the recursive formula for $x_{n}$ produces

$$
x_{n+1}=q^{n} \sum_{k=0}^{n}\left(\frac{1}{q^{3}}\right)^{k}= \begin{cases}n+1, & \text { if } q=1 \\ q^{n} \frac{1-\left(1 / q^{3}\right)^{n+1}}{1-1 / q^{3}}, & \text { if } q \neq 1\end{cases}
$$

Even in this case a number operator $\hat{N}$ can be introduced. It must satisfy the following relation $H_{o}=q^{\hat{N}-1} \frac{1-\left(1 / q^{3}\right)^{\hat{N}}}{1-1 / q^{3}}$. Such an operator satisfies again $\hat{N} \Phi_{j}=j \Phi_{j},[13$.

These conclusions can be easily generalized to different expressions of q-mutators which are not usually considered in the literature: in general, given $H_{O}$ satisfying the eigenvalue equation $H_{o} \Phi_{j}=x_{j} \Phi_{j}$, and introducing a map $X: \mathbb{N}_{0} \rightarrow \mathbb{R}$ such that, for each $m \in \mathbb{N}_{0} X(m)=x_{m}$, the number operator $\hat{N}$ is related to $H_{o}$ by $H_{o}=X(\hat{N})$, which, if $X$ is invertible, gives $\hat{N}$ as a function of $H_{o}: \hat{N}=X^{-1}\left(H_{o}\right)$.

Let us now introduce the following very general q-mutation relation:

$$
a a^{\dagger}-q a^{\dagger} a=f(q, \hat{N})
$$

where $f(q, \hat{N})$ is a self-adjoint operator depending on $q$ and $\hat{N}$ and such that $\lim _{q \rightarrow \pm 1} f(q, N)=1$ in some topological sense, for instance strongly on a dense domain. Using the spectral decomposition $f(q, \hat{N})=\sum_{j=0}^{\infty} f(q, j) P_{j}$, and repeating the same steps as before, we get the following expression for the $x_{n}$ 's:

$$
x_{n+1}=q^{n} f(q, 0)+q^{n-1} f(q, 1)+q^{n-2} f(q, 2)+\ldots+q f(q, n-1)+f(q, n)
$$

It is an easy exercise to check that this formula returns the results already obtained for the first and the second kind quons. Let us now consider some different examples. 
We start considering $f(q, \hat{N})=q^{-4 \hat{N}}$. In this case, assuming that $q \neq 1$, we obtain $x_{n}=$ $q^{n-1} \frac{1-\left(1 / q^{5}\right)^{n}}{1-1 / q^{5}}$. Again, if $q=1$ we get $x_{n}=n$. This is a natural extension of the result in (5.3) and the operator $\hat{N}$ satisfies the equality $H_{o}=q^{\hat{N}-1} \frac{1-\left(1 / q^{5}\right)^{\hat{N}}}{1-1 / q^{5}}$.

Let us now take $f(q, \hat{N})=e^{\left(q^{2}-1\right) \hat{N}}$. It is clear that $\lim _{q \rightarrow \pm 1} f(q, N)=1$. Applying formula (5.5) we get, once again $x_{n}=n$ if $q=1$, while, if $q \neq 1$, we obtain

$$
x_{n+1}=\frac{q^{n+1}-e^{(n+1)\left(q^{2}-1\right)}}{q-e^{q^{2}-1}},
$$

so that $\hat{N}$ must satisfies $H_{o}=\frac{q^{\hat{N}}-e^{\hat{N}\left(q^{2}-1\right)}}{q-e^{q^{2}-1}}$. Other examples can be easily constructed, but we will not do it here.

Summarizing, the so called quons appear just as particular cases of a much more general strategy, which consists in replacing the eigenvalues of the harmonic oscillator $\{n\}$ with a general non-negative sequence $\left\{x_{n}\right\}$ and the o.n. basis $\Phi_{n}=\frac{\left(a^{\dagger}\right)^{n}}{\sqrt{n !}} \Phi_{0}$ with an arbitrary o.n. basis of the Hilbert space. This is strongly related to what has been done in the rest of this paper. A deeper analysis of these aspects of the theory is in progress.

\section{Acknowledgements}

This work has been financially supported in part by M.U.R.S.T., within the project Problemi Matematici Non Lineari di Propagazione e Stabilità nei Modelli del Continuo, coordinated by Prof. T. Ruggeri.

\section{References}

[1] S.T. Ali, M. Engliš and J.-P. Gazeau, Vector coherent states from Plancherel's theorem, Clifford algebras and matrix domains, J. Phys. A37, (2004), 6067-6089.

[2] S.T. Ali, R. Roknizadeh and M.K. Tavassoly, Representations of coherent states in nonorthogonal bases, J. Phys. A37, (2004), 4407-4422.

[3] S.T. Ali, F. Bagarello, Some Physical Appearances of Vector Coherent States and CS Related to Degenerate Hamiltonians, J. Math. Phys, 46, 053518 (2005)

[4] S. T. Ali, J.-P. Antoine and J.-P. Gazeau, Coherent States, Wavelets and their Generalizations, Springer-Verlag, New York (2000) 
[5] F. Bagarello, Applications of Topological *-Algebras of Unbounded Operators, J. Math. Phys., 39, 2730-2747, (1998)

[6] F. Bagarello, C. Trapani, Algebraic dynamics in $O^{*}$-algebras: a perturbative approach, J. Math. Phys., 43, 3280-3292 (2002)

[7] F. Bagarello, A. Inoue, C Trapani, Derivations of quasi *-algebras, Int. Jour. Math. and Math. Sci., 21, 1077-1096 (2004)

[8] F. Bagarello, A. Inoue, C Trapani, Exponentiating derivations of quasi *algebras: possible approaches and applications, Int. Jour. Math. and Math. Sci., 17, 2805-2820 (2005)

[9] D.I. Fivel, Interpolation between Fermi and Bose statistics using generalized commutators, Phys. Rev. Lett., 65, 3361-3364, (1990); Erratum, Phys. Rev. Lett., 69, 2020, (1992); O.W. Greenberg, Particles with small violations of Fermi or Bose statistics, Phys. Rev. D, 43, 4111-4120, (1991); M. Bozejko, R. Speicher, An example of generalized Brownian motion, Comm. Math. Phys., 137, 519-531, (1991); P.E.T. Jorgensen, L. M. Schmitt, R. F. Werner, q-canonical commutation relations and stability of the Cuntz algebra, Pac. J. Math., 165, 131-151, (1994); D.A. Dubin, M.A. Hennings, A.I. Solomon, Integrable representations of the ultra-commutation relations, J. Math. Phys., 38, 3238-3262, (1997)

[10] J.P. Gazeau, J.R. Klauder, Coherent states for systems with discrete and continuous spectrum, J. Phys. A, 32, (1999), 123-132.

[11] J.R. Klauder, B.S. Skagerstam, Coherent states- Applications to physics and mathematical physics, World Scientific, Singapore (1985)

[12] G. Lassner, Topological algebras and their applications in Quantum Statistics, Wiss. Z. KMU-Leipzig, Math.-Naturwiss. R., 30, 572-595, (1981), Algebras of unbounded operators and quantum dynamics, Physica, 124 A, 471-480, (1984)

[13] R.N. Mohapatra, Infinite statistics and a possible small violation of the Pauli principle, Phys. Lett. B, 242, 407-411, (1990) 